\title{
Pharmacokinetic interactions between the potential COVID-19 treatment drugs lopinavir/ritonavir and arbidol in rats
}

\author{
Yunzhen $\mathrm{HU}^{1}$, Minjuan ZUO², Xiaojuan WANG ${ }^{1}$, Rongrong $\mathrm{WANG}^{1}$, Lu LI', Xiaoyang LU', \\ Saiping JIANG ${ }^{1 \bowtie}$ \\ ${ }^{I}$ Department of Clinical Pharmacy, the First Affiliated Hospital, Zhejiang University School of Medicine, Hangzhou 310006, China \\ ${ }^{2}$ Public Service Platform for the Evaluation of Innovative Drug Property, Hangzhou 311121, China
}

The coronavirus disease 2019 (COVID-19) pandemic caused by severe acute respiratory syndrome coronavirus 2 (SARS-CoV-2) has occasioned worldwide alarm. Globally, the number of reported confirmed cases has exceeded 84.3 million as of this writing (January 2, 2021). Since there are no targeted therapies for COVID-19, the current focus is the repurposing of drugs approved for other uses. In some clinical trials, antiviral drugs such as remdesivir (Grein et al., 2020), lopinavir/ritonavir (LPV/r) (Cao et al., 2020), chloroquine (Gao et al., 2020), hydroxychloroquine (Gautret et al., 2020), arbidol (Wang et al., 2020), and favipiravir (Cai et al., 2020b) have shown efficacy in COVID-19 patients. LPV/r combined with arbidol, which is the basic regimen in some regional hospitals in China including Zhejiiang Province, has shown antiviral effects in COVID-19 patients (Guo et al., 2020; Xu et al., 2020). A retrospective cohort study also reported that this combination therapy showed better efficacy than LPV/r alone for the treatment of COVID-19 patients (Deng et al., 2020).

According to the epidemiological studies, $14 \%$ $53 \%$ of COVID-19 patients showed abnormal levels of serum aminotransferases during disease progression, which could have been caused by viral infection of liver cells or drug-induced hepatotoxicity (Zhang et al., 2020). There was a significantly higher proportion of patients with abnormal liver function after treatment

$\triangle$ Saiping JIANG,j5145@zju.edu.cn

(D) Saiping JIANG, https://orcid.org/0000-0001-7720-2725

Received Jan. 3, 2021; Revision accepted Feb. 21, 2021; Crosschecked May 12, 2021

(C) Zhejiang University Press 2021 with LPV/r (Cai et al., 2020a). As arbidol produced similar adverse reactions, the combination of these two drugs may increase the risk of liver injury. In our retrospective observational study, 76 of 131 adult COVID-19 patients developed liver injury, and the combination of LPV/r with arbidol was a risk factor for liver injury in non-critical COVID-19 patients (Jiang et al., 2020).

In the $\mathrm{LPV} / \mathrm{r}$ regimen, lopinavir is combined with a low dose of ritonavir, which functions as a protease inhibitor. The bioavailability of lopinavir is relatively poor, characterized by rapid and extensive metabolism. The sub-therapeutic dose of ritonavir is used as a potent cytochrome P450 3A4 (CYP3A4) inhibitor to suppress metabolism of lopinavir and boost its plasma concentration, thus increasing its efficacy. Both lopinavir and ritonavir have shown hepatotoxicity in the clinic, producing moderate to severe elevations of serum aminotransferase levels in $3 \%-10 \%$ and $15 \%$ of patients, respectively (LiverTox, 2012). Arbidol may also elevate serum aminotransferases, but the exact proportion of patients that occurs has not been reported. Given that CYP3A4 is a major isoenzyme involved in the metabolism of arbidol, interactions between LPV/r and arbidol, leading to increased exposure to LPV/r or arbidol, may be expected. Therefore, this interaction may imply a higher risk of hepatotoxicity. Taking these facts into consideration, we performed a study on the pharmacokinetic interactions between LPV/r and arbidol in order to provide some initial guidelines for combined therapy with these two drugs.

We established a rat model to investigate the pharmacokinetic interactions between LPV/r and arbidol. Rats were divided into three groups (arbidol, 
$\mathrm{LPV} / \mathrm{r}$, and combination), each consisting of three male and three female rats. Arbidol was administered by gavage at a dose of $25 \mathrm{mg} / \mathrm{kg}$, and LPV/r was composed of $50 \mathrm{mg} / \mathrm{kg}$ lopinavir and $12.5 \mathrm{mg} / \mathrm{kg}$ ritonavir. The concentrations of arbidol and lopinavir were determined by liquid chromatography-tandem mass spectrometry (LC-MS/MS).

As shown in Table 1 and Fig. 1, when co-administered with $\mathrm{LPV} / \mathrm{r}$, the maximum concentration $\left(C_{\max }\right)$ of arbidol increased from $(129.8 \pm 99.0)$ to $(221.8 \pm 112.1) \mathrm{ng} / \mathrm{mL}$ $(P>0.05)$, and the area under the plasma concentrationtime curve from time zero to infinity $\left(\mathrm{AUC}_{0-\infty}\right)$ increased from $(705.6 \pm 258.5)$ to $(1250.3 \pm 255.5) \mathrm{h} \cdot \mathrm{ng} / \mathrm{mL}(P<0.01)$. The pharmacokinetics of arbidol differed between male and female animals. In the arbidol group, the mean $C_{\max }$ and $\mathrm{AUC}_{0-\infty}$ of arbidol were 3.0 and 1.8 times higher in female rats than in male rats, respectively. In the combination group, the mean $C_{\max }$ and $\mathrm{AUC}_{0-\infty}$ of arbidol were 2.6 and 1.2 times higher in female rats than in male rats, respectively.

As shown in Table 2 and Fig. 2, when co-administered with arbidol, the $C_{\max }$ of lopinavir increased from $(2336.4 \pm 504.7)$ to $(3500.6 \pm 749.6) \mathrm{ng} / \mathrm{mL}(P<0.05)$, and the $\mathrm{AUC}_{0-\infty}$ increased from $(27985.1 \pm 11314.6)$ to (37 101.8 \pm 10 083.6) h·ng/mL $(P>0.05)$. The pharmacokinetic parameters of lopinavir were also influenced by the sex of the experimental animals. In the $\mathrm{LPV} / \mathrm{r}$ and combination groups, the mean $\mathrm{AUC}_{0-\infty}$ of lopinavir was 1.9 and 1.4 times higher in female rats than in male rats, respectively, and the time to maximum concentration $\left(t_{\max }\right)$ was also longer in female rats than in male rats.

Combined antiviral therapy may be an effective approach for curing patients infected with SARS-CoV-2. $\mathrm{LPV} / \mathrm{r}$ in combination with arbidol is the mainstay of treatment in Zhejiang Province. However, not enough attention has been paid to the safety of these combination drug therapies. Based on clinical observations, the combination of these two drugs may increase the risk of liver injury. Due to their metabolic characteristics, potential drug-drug interactions must be taken into
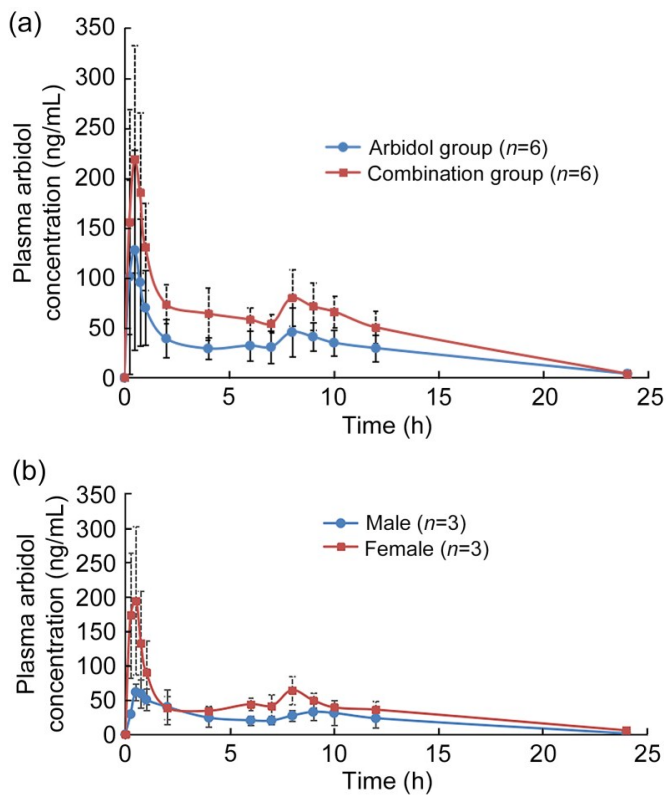

(c)

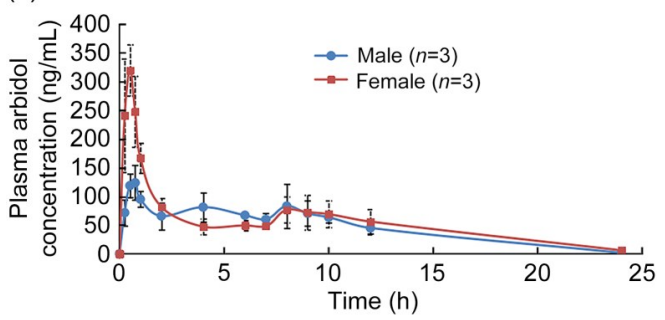

Fig. 1 Plasma concentration-time courses of arbidol: (a) in the presence and absence of $\mathrm{LPV} / \mathrm{r}$; (b) male and female rats in the arbidol group; (c) male and female rats in the combination group. Data are expressed as mean \pm standard deviation (SD). LPV/r: lopinavir/ritonavir.

Table 1 Pharmacokinetic parameters of arbidol after oral administration of arbidol or co-administration of arbidol and $\mathbf{L P V} / \mathbf{r}$ in rats

\begin{tabular}{lccccc}
\hline \multicolumn{1}{c}{ Group } & $t_{1 / 2}(\mathrm{~h})$ & $t_{\max }(\mathrm{h})$ & $C_{\max }(\mathrm{ng} / \mathrm{mL})$ & $\mathrm{AUC}_{0-t}(\mathrm{~h} \cdot \mathrm{ng} / \mathrm{mL})$ & $\mathrm{AUC}_{0-\infty}(\mathrm{h} \cdot \mathrm{ng} / \mathrm{mL})$ \\
\hline Arbidol & & & & & \\
$\quad$ Male $(n=3)$ & $2.7 \pm 0.4$ & $0.6 \pm 0.1$ & $65.2 \pm 16.8$ & $479.0 \pm 122.6$ & $497.3 \pm 116.8$ \\
Female $(n=3)$ & $4.2 \pm 1.5$ & $0.5 \pm 0.0$ & $194.3 \pm 108.1$ & $872.1 \pm 166.5$ & $913.9 \pm 152.4$ \\
$\quad$ Total $(n=6)$ & $3.5 \pm 1.3$ & $0.5 \pm 0.1$ & $129.8 \pm 99.0$ & $675.6 \pm 251.9^{* *}$ & $705.6 \pm 258.5^{* *}$ \\
Combination & & & & & \\
$\quad$ Male $(n=3)$ & $2.7 \pm 0.0$ & $0.7 \pm 0.1$ & $124.3 \pm 29.3$ & $1133.9 \pm 206.3$ & $1141.0 \pm 207.1$ \\
Female $(n=3)$ & $3.6 \pm 0.6$ & $0.5 \pm 0.0$ & $319.4 \pm 44.8$ & $1326.2 \pm 267.9$ & $1359.7 \pm 290.7$ \\
Total $(n=6)$ & $3.2 \pm 0.6$ & $0.6 \pm 0.1$ & $221.8 \pm 112.1$ & $1230.0 \pm 238.4$ & $1250.3 \pm 255.5$ \\
\hline
\end{tabular}

Data are expressed as mean \pm standard deviation (SD). ${ }^{* *} P<0.01$, compared with those in the combination group. LPV/r: lopinavir/ritonavir; $t_{1 / 2}$ : half-life; $t_{\max }$ : time to maximum concentration; $C_{\max }$ : maximum concentration; $\mathrm{AUC}_{0-t}$ : area under the plasma concentration-time curve from time zero to the last quantifiable time point; $\mathrm{AUC}_{0-\infty}$ : area under the plasma concentration-time curve from time zero to infinity. 
Table 2 Pharmacokinetic parameters of lopinavir after oral administration of LPV/r or co-administration of LPV/r and arbidol in rats

\begin{tabular}{lrrrll}
\hline \multicolumn{1}{c}{ Group } & \multicolumn{1}{c}{$t_{1 / 2}(\mathrm{~h})$} & \multicolumn{1}{c}{$t_{\max }(\mathrm{h})$} & \multicolumn{1}{c}{$C_{\max }(\mathrm{ng} / \mathrm{mL})$} & $\mathrm{AUC}_{0-t}(\mathrm{~h} \cdot \mathrm{ng} / \mathrm{mL})$ & $\mathrm{AUC} C_{0-\infty}(\mathrm{h} \cdot \mathrm{ng} / \mathrm{mL})$ \\
\hline $\mathrm{LPV} / \mathrm{r}$ & & & & & \\
$\quad$ Male $(n=3)$ & $1.5 \pm 0.5$ & $6.0 \pm 2.0$ & $2098.0 \pm 434.8$ & $18142.9 \pm 3955.2$ & $19042.7 \pm 4621.6$ \\
Female $(n=3)$ & $3.5 \pm 1.8$ & $10.0 \pm 2.0$ & $2574.8 \pm 526.5$ & $33196.4 \pm 12906.2$ & $36927.5 \pm 7667.8$ \\
$\quad$ Total $(n=6)$ & $2.5 \pm 1.6$ & $8.0 \pm 2.8$ & $2336.4 \pm 504.7^{*}$ & $25669.6 \pm 11868.8$ & $27985.1 \pm 11314.6$ \\
Combination & & & & & \\
Male $(n=3)$ & $2.0 \pm 0.3$ & $6.3 \pm 0.6$ & $3565.8 \pm 943.3$ & $28453.9 \pm 6323.4$ & $30438.2 \pm 7227.4$ \\
Female $(n=3)$ & $1.9 \pm 0.4$ & $9.7 \pm 0.6$ & $3435.4 \pm 708.7$ & $43657.0 \pm 8424.1$ & $43765.4 \pm 8291.4$ \\
Total $(n=6)$ & $2.0 \pm 0.4$ & $8.0 \pm 1.9$ & $3500.6 \pm 749.6$ & $36055.4 \pm 10664.0$ & $37101.8 \pm 10083.6$ \\
\hline
\end{tabular}

Data are expressed as mean \pm standard deviation (SD). ${ }^{*} P<0.05$, compared with those in the combination group. LPV/r: lopinavir/ritonavir; $t_{1 / 2}$ : half-life; $t_{\max }$ : time to maximum concentration; $C_{\max }$ : maximum concentration; $\mathrm{AUC}_{0-t}$ : area under the curve from time zero to the last quantifiable time point; $\mathrm{AUC}_{0-\infty}$ : area under the curve from time zero to infinite.
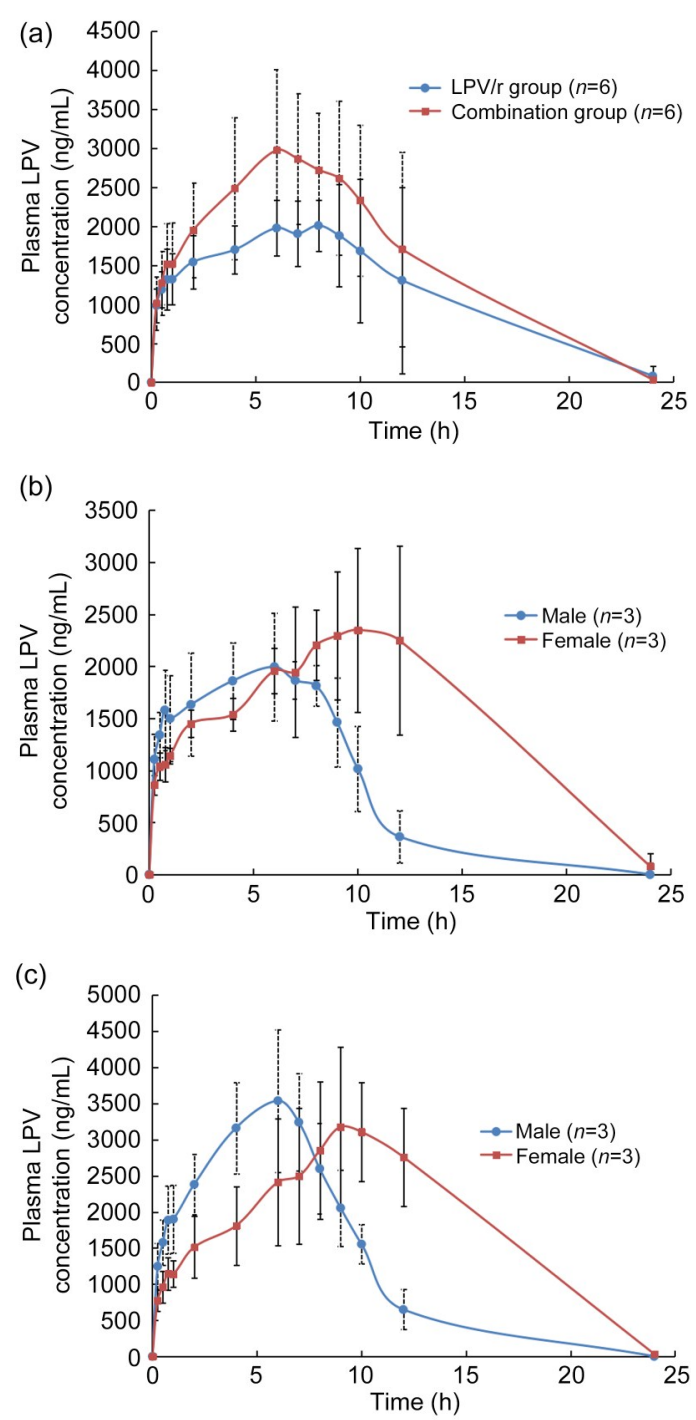

Fig. 2 Plasma concentration-time courses of lopinavir: (a) in the presence and absence of arbidol; (b) male and female rats in the LPV/r group; (c) male and female rats in the combination group. Data are expressed as mean \pm standard deviation (SD). LPV/r: lopinavir/ritonavir. account. Our study is the first to evaluate the pharmacokinetic interactions between LPV/r and arbidol in rats. When combined with LPV/r, exposure to arbidol increased acutely by approximately two times. Meanwhile, the $C_{\max }$ of lopinavir increased significantly (1.5 times). Since the pharmacokinetic profile of arbidol is influenced by CYP3A4, one can deduce that drug-drug interactions will change its plasma concentrations. In our study, given the role played by CYP3A4 inhibition, LPV/r increased the bioavailability of arbidol, while arbidol had no obvious effect on the AUC of lopinavir in the presence of ritonavir.

A significant, albeit accidental, finding of our study was that the pharmacokinetic profiles of arbidol and lopinavir differed depending on the gender of the experimental animals. Since arbidol was more sensitive to this gender effect, this may influence its safety and efficacy. Gender-based differences in CYP3A expression in rats are likely to explain these observations. This pharmacokinetic characteristic of aribidol has never been reported before. In the case of lopinavir, several clinical studies with patients did not show obvious differences in plasma concentrations between sexes (Ofotokun et al., 2007; Umeh et al., 2011). In contrast, Jullien et al. (2006) reported that clearance/ bioavailability $(\mathrm{CL} / \mathrm{F})$ of lopinavir was gender-related, with a $39 \%$ increase observed in males compared with females.

We acknowledge that our study has some limitations. Firstly, both LPV/r and arbidol were given in a single dose. In clinical practice, arbidol is administered three times a day and LPV/r twice a day. Hence, multiple doses may cause more accumulation of arbidol and lopinavir, and this cannot be ignored. Secondly, this study involved animal experiments and our conclusions have yet to be confirmed in clinical trials. Thirdly, the association between increased 
arbidol exposure, lopinavir $C_{\max }$, and exacerbated drug-induced liver injury should be studied further. Finally, only three rats were used to compare the pharmacokinetic differences between males and females, which is not sufficient to produce statistically significant results. Therefore, the findings reported here should be considered preliminary.

In summary, combined administration of LPV/r and arbidol produced a significant increase in the AUC of arbidol and the $C_{\max }$ of lopinavir. The interaction between these two drugs may affect the safety of the combined treatment. Therefore, appropriate dose adjustment is recommended when LPV/r and arbidol are administered concurrently, and the dose of arbidol may need to be halved. The results obtained in this study may be useful to predict interactions between LPV/r and other medications metabolized by CYP3A4.

\section{Acknowledgments}

This work is supported by the National Natural Science Foundation of China (No. 81703612).

\section{Author contributions}

Yunzhen HU, Saiping JIANG, and Xiaoyang LU conceived and designed this study. Minjuan ZUO and Xiaojuan WANG carried out the experiments. Yunzhen HU performed data analysis and drafted the manuscript. Rongrong WANG and $\mathrm{Lu} \mathrm{LI}$ made critical contribution to the discussion. All authors have read and approved the final manuscript and, therefore, have full access to all the data in the study and take responsibility for the integrity and security of the data.

\section{Compliance with ethics guidelines}

Yunzhen HU, Minjuan ZUO, Xiaojuan WANG, Rongrong WANG, Lu LI, Xiaoyang LU, and Saiping JIANG declare that they have no conflict of interest.

The Research Ethics Committee of the First Affiliated Hospital, Zhejiang University School of Medicine (Hangzhou, China) approved the protocol followed in this study. All institutional and national guidelines for the care and use of laboratory animals were followed.

\section{References}

Cai QX, Huang DL, Yu H, et al., 2020a. COVID-19: abnormal liver function tests. J Hepatol, 73(3):566-574. https://doi.org/10.1016/j.jhep.2020.04.006

Cai QX, Yang MH, Liu DJ, et al., 2020b. Experimental treatment with favipiravir for COVID-19: an open-label control study. Engineering (Beijing), 6(10):1192-1198. https://doi.org/10.1016/j.eng.2020.03.007

Cao B, Wang YM, Wen DN, et al., 2020. A trial of lopinavirritonavir in adults hospitalized with severe Covid-19. N Engl J Med, 382(19):1787-1799.

https://doi.org/10.1056/NEJMoa2001282
Deng LS, Li CN, Zeng Q, et al., 2020. Arbidol combined with $\mathrm{LPV} / \mathrm{r}$ versus $\mathrm{LPV} / \mathrm{r}$ alone against corona virus disease 2019: a retrospective cohort study. J Infect, 81(1):E1-E5. https://doi.org/10.1016/j.jinf.2020.03.002

Gao JJ, Tian ZX, Yang X, 2020. Breakthrough: chloroquine phosphate has shown apparent efficacy in treatment of COVID-19 associated pneumonia in clinical studies. Biosci Trends, 14(1):72-73. https://doi.org/10.5582/bst.2020.01047

Gautret P, Lagier JC, Parola P, et al., 2020. Hydroxychloroquine and azithromycin as a treatment of COVID-19: results of an open-label non-randomized clinical trial. Int $J$ Antimicrob Agents, 56(1):105949. https://doi.org/10.1016/j.ijantimicag.2020.105949

Grein J, Ohmagari N, Shin D, et al., 2020. Compassionate use of remdesivir for patients with severe Covid-19. N Engl J Med, 382(24):2327-2336. https://doi.org/10.1056/NEJMoa2007016

Guo YZ, Xu KJ, Li YT, et al., 2020. Safety of protease inhibitors and Arbidol for SARS-CoV-2 pneumonia in Zhejiang Province, China. $J$ Zhejiang Univ-Sci B (Biomed \& Biotechnol), 21(12):948-954. https://doi.org/10.1631/jzus.B2000204

Jiang SP, Wang RR, Li L, et al., 2020. Liver injury in critically ill and non-critically ill COVID-19 patients: a multicenter, retrospective, observational study. Front Med (Lausanne), 7:347. https://doi.org/10.3389/fmed.2020.00347

Jullien V, Urien S, Hirt D, et al., 2006. Population analysis of weight-, age-, and sex-related differences in the pharmacokinetics of lopinavir in children from birth to 18 years. Antimicrob Agents Chemother, 50(11):3548-3555. https://doi.org/10.1128/AAC.00943-05

LiverTox, 2012. Clinical and research information on druginduced liver injury: protease inhibitors (HIV). Bethesda (MD): National Institute of Diabetes and Digestive and Kidney Diseases. https://www. ncbi. nlm. nih. gov/books/ NBK548893

Ofotokun I, Chuck SK, Binongo JN, et al., 2007. Lopinavir/ ritonavir pharmacokinetic profile: impact of sex and other covariates following a change from twice-daily to oncedaily therapy. J Clin Pharmacol, 47(8):970-977. https://doi.org/10.1177/0091270007302564

Umeh OC, Currier JS, Park JG, et al., 2011. Sex differences in lopinavir and ritonavir pharmacokinetics among HIVinfected women and men. J Clin Pharmacol, 51(12): 1665-1673. https://doi.org/10.1177/0091270010388650

Wang ZL, Yang BH, Li QW, et al., 2020. Clinical features of 69 cases with coronavirus disease 2019 in Wuhan, China. Clin Infect Dis, 71(15):769-777. https://doi.org/10.1093/cid/ciaa272

Xu KJ, Cai HL, Shen YH, et al., 2020. Management of COVID19: the Zhejiang experience. J Zhejiang Univ (Med Sci), 49(2):147-157 (in Chinese). https://doi.org/10.3785/j.issn.1008-9292.2020.02.02

Zhang C, Shi L, Wang FS, 2020. Liver injury in COVID-19: management and challenges. Lancet Gastroenterol Hepatol, 5(5):428-430.

https://doi.org/10.1016/S2468-1253(20)30057-1 